\title{
SIMULAÇÃO E ANÁLISE DO EFEITO DA PROPORÇÃO DE REAGENTES NA POLIMERIZAÇÃO RADICALAR POR TRANSFERÊNCIA DE ÁTOMO
}

\author{
R. P. VIEIRA ${ }^{1}$, A. PERES ${ }^{2}$, J. M. COSTA ${ }^{2}$, L. M. F. LONA ${ }^{1}$ \\ ${ }^{1}$ Universidade Estadual de Campinas, Faculdade de Engenharia Química \\ ${ }^{2}$ Petrobras, Centro de Pesquisa e Desenvolvimento \\ e-mail para contato: liliane@ feq.unicamp.br
}

\begin{abstract}
RESUMO - A síntese de materiais poliméricos via ATRP (polimerização radicalar por transferência de átomo) possibilita agregar propriedades requisitadas em diversos ramos industriais (indústria automobilística, tintas, embalagens, adesivos, saúde e beleza etc.). Tendo em vista a importância de tal processo e a versatilidade em estudá-lo por meio de simulações, o presente trabalho expõe uma análise da influência da proporção dos reagentes na taxa de polimerização e nas propriedades finais do polímero formado através de simulações. Avaliou-se a influência de duas proporções iniciais de reagentes ([iniciador $]_{0} /[\text { catalisador }]_{0}=$ $0,1$ e [iniciador $\left.]_{0} /[\text { catalisador }]_{0}=10\right)$. Os resultados mostraram que há igual aumento na taxa de polimerização à medida que se alteram as concentrações de reagentes nessas proporções. Porém, as polidispersidades não apresentaram grande variação, sugerindo que tal análise teórica pode ser utilizada como ferramenta para a otimização do tempo reacional a nível experimental.
\end{abstract}

\section{INTRODUÇÃO}

A polimerização radicalar via transferência de átomo (ATRP) consiste numa das técnicas de polimerização radicalar controlada para a síntese de materiais com estruturas macromoleculares específicas. Tais propriedades são obtidas em virtude da conformação macromolecular dos polímeros formados, que podem ser homopolímeros monodispersos (baixo índice de polidispersidade) (Zhao et al., 2013), copolímeros em bloco (Porras et al., 2013), em gradiente (Zhou et al., 2012) e polímeros funcionalizados (Badri et al., 2012; Goldmann et al., 2013; Salian e Byrne, 2013). Estes materiais com diferentes estruturas encontram aplicações em diversas áreas, como na indústria automotiva e aeroespacial (Salzinger e Rieger, 2012), cosméticos (Pham et al., 2013), na indústria médica e farmacêutica (Destarac, 2010; Singh et al., 2012).

Dentre as técnicas de polimerização controlada, a ATRP consiste na mais promissora pelo fato de poder ser utilizada para vários tipos de monômeros, requerer condições de polimerização em temperaturas brandas e ser extremamente robusta com relação às impurezas (Matyjaszewski, 2012). Apesar de o processo ATRP ter recebido uma grande atenção nos últimos anos, uma quantidade bem reduzida de trabalhos focou o desenvolvimento de modelos matemáticos e simulações para representa-lo; como exemplos, encontram destaque pesquisas com modelagem cinética, modelos para reatores batelada, semibatelada e CSTR, 
além de modelos utilizando iniciadores bifuncionais (Al-harthi et al., 2007; Zhang e Ray, 2002; Zhu, 1999); Mais recentemente, tem-se o trabalho de Vieira et al., no qual desenvolveram um modelo utilizando o método dos momentos, que possibilitou prever os perfis de concentração dos reagentes e as propriedades médias dos polímeros (Massas molares e polidispersidades) (Vieira et al., 2013).

Cada um dos trabalhos supracitados possuem contribuições particulares por meio da análise teórica obtida através das simulações. Porém, nenhum desses trabalhos utilizou a técnica de simulação como análise da concentração inicial dos reagentes. Este fato é intrigante, uma vez que é amplamente conhecida a importância da proporção de reagentes no estudo cinético de qualquer sistema reacional. Em nível experimental, os diversos trabalhos envolvendo ATRP utilizam proporções iguais de iniciador e catalisador, como via de regra, para qualquer sistema. A questão é: até que ponto a proporção de reagentes influencia o processo ATRP?

O objetivo deste trabalho é explorar a influência das concentrações iniciais de catalisador e iniciador na cinética (conversão de monômero) e nas propriedades (massas molares e polidispersidades) do poliestireno formado em um reator batelada isotérmico através de simulações utilizando um modelo matemático baseado no método dos momentos.

\section{METODOLOGIA}

O desenvolvimento das simulações deste trabalho tem por base o modelo matemático do trabalho de Vieira et al. (2013). Em tal pesquisa os autores expõem o método de obtenção de um sistema de equações diferenciais ordinárias (EDO's). A resolução numérica deste sistema proporciona a obtenção do perfil de concentração de monômero e dos perfis de momentos populacionais (Ray, 1972), que permitem determinar as propriedades médias do polímero formado (massas molares e polidispersidades). Para a solução do sistema de EDO's, foi desenvolvido um programa computacional em linguagem Fortran, com o auxílio da subrotina LSODE (Hindmarsh, 1983). Esta sub-rotina utiliza o método de Adams-Moulton para resolver problemas de valores iniciais com sistemas de equações diferenciais rígidas.

\subsection{Dados de entrada do programa computacional}

Os dados de entrada do programa computacional consistem nas concentrações iniciais dos reagentes utilizados e parâmetros cinéticos que, para o estudo de caso deste trabalho, foram obtidos da pesquisa de $\mathrm{Fu}$ et al. (2007). A parte experimental considerou uma polimerização em massa a $110{ }^{\circ} \mathrm{C}$, com 2-bromopropanoato de metila (abreviação usual $\mathrm{MBrP}$ ) como iniciador e brometo de cobre (I) (CuBr) complexado em N,N,N',N",N"pentametildietilenotriamina (PMDETA) como sistema catalítico (Fu et al., 2007).

A primeira simulação realizada objetivou reproduzir o trabalho experimental de $\mathrm{Fu}$ et al. (2007), no qual as concentrações iniciais de monômero, iniciador, catalisador e ligante estiveram numa proporção de 50:1:1:1, sendo que $[\mathrm{M}]_{0}=15 \mathrm{~mol} \mathrm{~L}^{-1}$. Para a análise proposta nesta pesquisa, realizaram-se mais duas simulações utilizando proporções diferentes de iniciador e catalisador. Assim, três razões de concentrações são avaliadas: $[\mathrm{RX}]_{0} /[\mathrm{C}]_{0}=0,1$; $[\mathrm{RX}]_{0} /[\mathrm{C}]_{0}=1$ (FU et al., 2007) e $[\mathrm{RX}]_{0} /[\mathrm{C}]_{0}=10$. 


\subsection{Dados de saída do programa computacional}

Dispondo das concentrações iniciais de reagentes e dos parâmetros cinéticos do processo, obtêm-se os dados de saída do programa computacional (massas molares, polidisersidades e conversão de monômero). A solução do sistema de EDO's proposta no trabalho de Vieira et al. (2013) possibilitou a obtenção de propriedades médias extremamente importantes dos polímeros, tais como massa molar média numérica $(\overline{M n})$, massa molar média ponderal $(\overline{M w})$, índice de polidispersidade (IPD), além da conversão de monômero em cada instante da reação. Para a obtenção da massa molar média numérica, foi necessário o valor dos momentos de ordem "zero" e "um" de cada espécie envolvida, como definido por Ray (1972), na Equação 1.

$$
\overline{M n}=M W_{M}\left(\frac{\mu_{1}+\lambda_{11}+\lambda_{12}+\delta_{1}}{\mu_{0}+\lambda_{01}+\lambda_{02}+\delta_{0}}\right)
$$

Na qual $\overline{M n}$ é a massa molar média numérica do polímero e $M W_{M}$ é a massa molar de monômero. Em paralelo a isto, a obtenção da massa molar média ponderal levou em conta os momentos de ordem "um" e "dois" de cada espécie envolvida, como descrito na Equação 2 .

$$
\overline{M w}=M W_{M}\left(\frac{\mu_{2}+\lambda_{21}+\lambda_{22}+\delta_{2}}{\mu_{1}+\lambda_{11}+\lambda_{12}+\delta_{1}}\right)
$$

Na qual $\overline{M w}$ é a massa molar média ponderal do polímero e $M W_{M}$ é a massa molar do monômero. Após a obtenção de $\overline{M n}$ e $\overline{M w}$, o índice de polidispersidade do polímero (IPD) foi obtido através da Equação 3.

$$
I P D=\frac{\overline{M w}}{\overline{M n}}
$$

Por fim, a conversão de monômero com o tempo de reação foi obtida através da Equação 4.

$$
\text { conversão }=1-\frac{[M]}{[M]_{0}}
$$

Em que $[M]$ é a concentração de monômero em função do tempo e $[M]_{0}$ é a concentração inicial de monômero. 


\section{RESULTADOS E DISCUSSÃO}

A Figura 1 fornece o resultado da influência da proporção de reagentes na evolução da conversão de monômero com tempo.

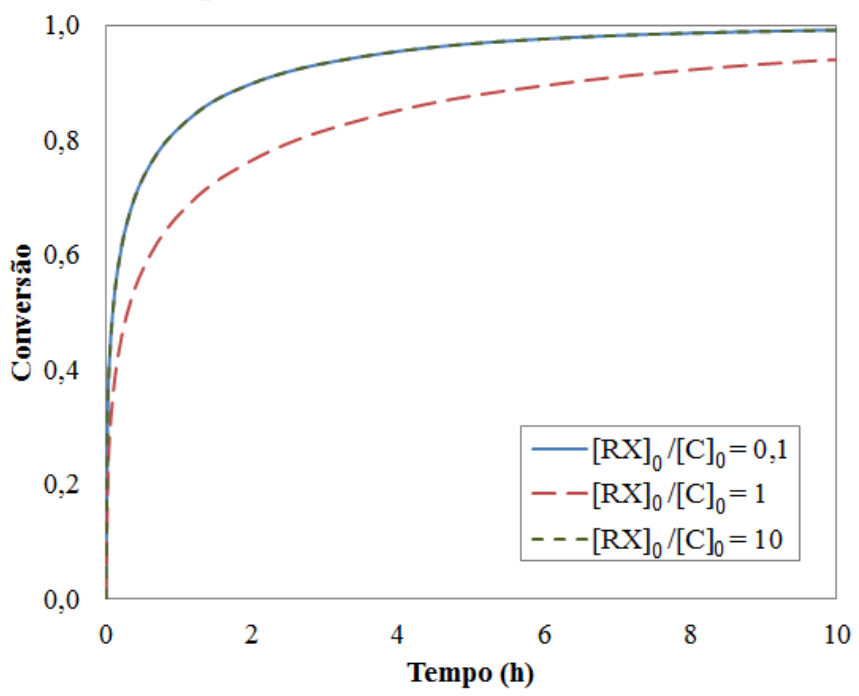

Figura 1 - Influência da relação entre as concentrações iniciais de iniciador e catalisador na conversão de monômero.

Analisando-se a Figura, percebe-se um aumento na conversão de monômero por meio da alteração na razão $[\mathrm{RX}]_{0} /[\mathrm{C}]_{0}$. Para ambos os casos em que a relação foi alterada em dez vezes, a conversão de monômero permaneceu exatamente igual, ou seja, as curvas para $[\mathrm{RX}]_{0} /[\mathrm{C}]_{0}=0,1 \mathrm{e}[\mathrm{RX}]_{0} /[\mathrm{C}]_{0}=10$ são coincidentes. Este resultado indica que o aumento na concentração inicial de cada um desses reagentes, de forma isolada, proporciona um aumento igual na taxa de polimerização. A explicação para este fato encontra-se no estudo da cinética de polimerização (Tang e Matyjaszewski, 2007). Em tal estudo, os autores determinaram constantes cinéticas de ativação do processo para vários iniciadores, juntamente com a influência de sua concentração. Com o aumento de dez vezes na concentração inicial de iniciador, uma grande quantidade de radicais é formado nos primeiros instantes da polimerização, fazendo com que a propagação também aumente. Isto ocorre pelo fato da taxa de polimerização ser proporcional à concentração de radicais em propagação (Equação 5).

$$
R_{p}=k_{p}[M] \sum_{n=1}^{\infty}\left[P_{n}^{\bullet}\right]
$$

Por outro lado, aumentando-se a concentração de catalisador na mesma proporção, observa-se um aumento exatamente igual na taxa de polimerização, confirmando o que foi observado experimentalmente (Ohno et al., 1998). Junto a isto, uma análise da Figura 2 permite confirmar que um aumento na concentração de $\mathrm{CuBr}(\mathrm{I}) / \mathrm{PMDETA}$ faz com que o equilíbrio químico seja deslocado de modo a ativar as espécies dormentes, ou seja, uma maior quantidade de radicais poliméricos se tornam em propagação, ocasionando um aumento na taxa. 


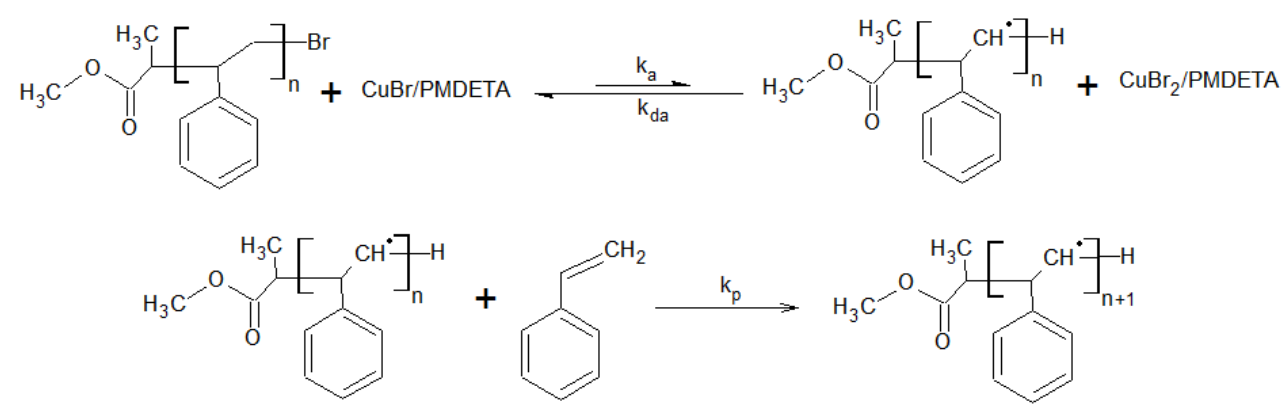

Figura 2 - Mecanismo reacional da etapa de propagação das cadeias poliméricas na polimerização radicalar via transferência de átomo do estireno iniciado por $\mathrm{MBrP}$, utilizando $\mathrm{CuBr} / \mathrm{PMDETA}$ como sistema catalítico.

A Figura 3 fornece a influência da relação entre as concentrações de iniciador e catalisador na evolução da massa molar média numérica.

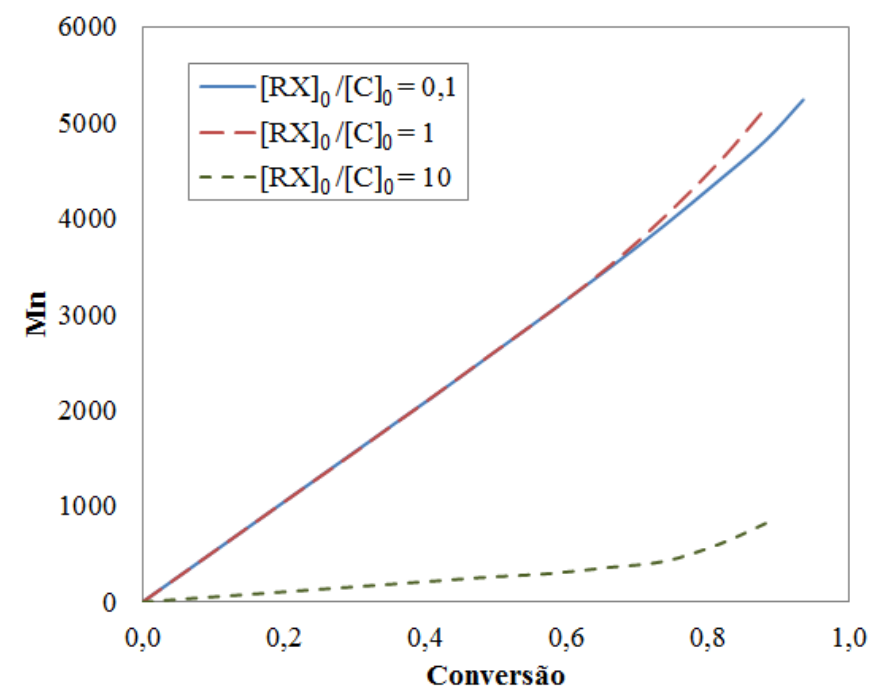

Figura 3 - Influência da relação entre as concentrações de iniciador e catalisador na evolução da massa molar média numérica em função da conversão de monômero.

Através da Figura 3 é possível observar que o aumento, em dez vezes, da concentração de catalisador $\left([\mathrm{RX}]_{0} /[\mathrm{C}]_{0}=0,1\right)$ não apresenta influência significativa na evolução de massa molar, se comparada com uma razão equimolar entre esses reagentes. Por outro lado, aumentando-se a concentração de iniciador em dez vezes $\left([\mathrm{RX}]_{0} /[\mathrm{C}]_{0}=10\right)$, percebe-se que há uma drástica redução da massa molar. Este resultado está, obviamente, ligado ao fato da produção de uma grande quantidade de radicais primários no início do processo. Esses radicais, por sua vez, iniciam a propagação através da reação com uma maior quantidade de monômeros do que aquela em $[\mathrm{RX}]_{0} /[\mathrm{C}]_{0}=1$, havendo, assim, uma maior concentração de polímeros com baixíssimas massas molares $(\mathrm{Mn}<1000)$. A Figura 4 fornece a influência da relação entre as concentrações de iniciador e catalisador no índice de polidispersidade do polímero formado. 


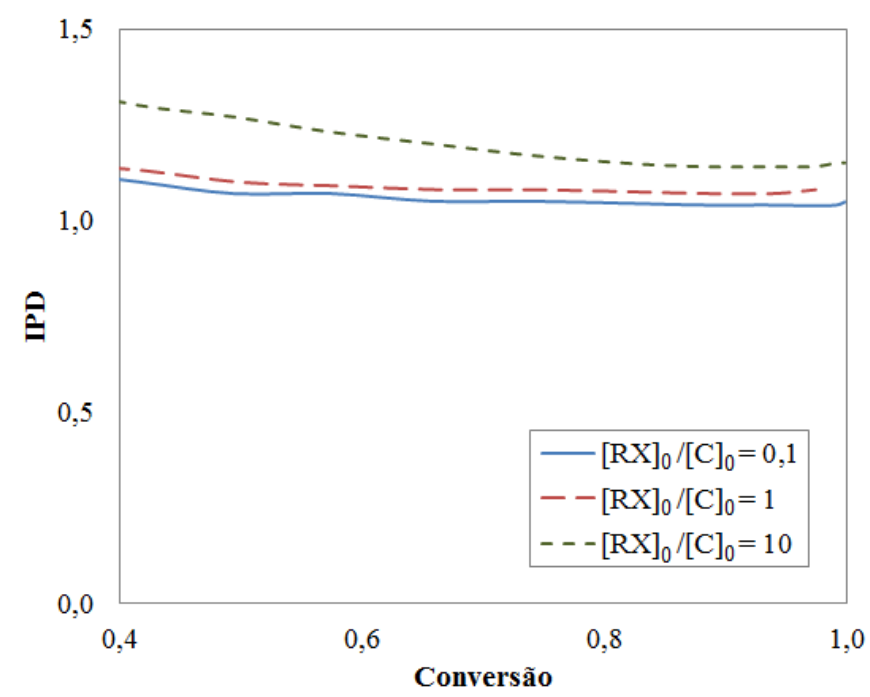

Figura 4 - Influência da relação entre as concentrações de iniciador e catalisador no índice de polidispersidade do polímero formado.

A Figura 4 revela que a variação em dez vezes na relação entre as concentrações de iniciador e catalisador não alteram drasticamente o índice de polidispersidade do polímero formado. O controle proporcionado pelo sistema reacional escolhido ainda continua bastante eficiente (todos os IPDs são menores do que 1,5). Porém, nota-se que existe uma pequena diferença entre esses valores. Essa diferença pode ser explicada através da Equação 6, derivada do estudo cinético do processo ATRP (Tang e Matyjaszewski, 2007).

$$
I P D=1+\frac{k_{p}[R X]_{0}}{k_{d a}[C u(I I)]}\left(\frac{2}{\text { Conversão }}-1\right)
$$

Uma grande concentração inicial de iniciador faz com que o numerador do segundo termo da Equação 6 seja maior, ocasionando um maior IPD, como ocorre no caso em que $[\mathrm{RX}]_{0} /[\mathrm{C}]_{0}=10$. O oposto disso é válido para um menor índice de polidispersidade em $[\mathrm{RX}]_{0} /[\mathrm{C}]_{0}=0,1$. Junto a isto, atribui-se essa diferença, também, à concentração do desativador $\mathrm{Cu}(\mathrm{II})$ proveniente da oxidação do catalisador $(\mathrm{CuBr})$. Uma maior concentração de catalisador faz com que o equilíbrio seja deslocado no sentido de formar espécies em propagação e $\mathrm{CuBr}_{2}$ (Figura 2). Essas espécies encontram-se no denominador da Equação 6. Portanto, quanto maior a concentração de desativador no sistema, menor o índice de polidispersidade, como está ilustrado na Figura 4. Apesar da variação entre as concentrações de catalisador e iniciador terem sido aumentadas em dez vezes, o que é bastante significativo, observa-se que não há uma drástica variação nos índices de polidispersidades da Figura 4.

\section{CONCLUSÕES}

A variação da proporção de reagentes não apresenta influência direta sobre a polidispersidade do material formado. Isto justifica a maior parte dos trabalhos experimentais utilizar a mesma proporção de reagentes. Entretanto, quando a questão está na produção de materiais controlados a nível industrial, o tempo reacional é um fator crucial para o design do reator. $\mathrm{O}$ resultado proposto neste trabalho, referente à taxa de polimerização, sugere que o 
aumento da concentração de catalisador promove um progressivo aumento na taxa de polimerização sem alterar as propriedades finais do polímero formado (a razão $[\mathrm{RX}]_{0} /[\mathrm{C}]_{0}=$ 0,1 aumenta a taxa de polimerização, reduz a polidispersidade e não altera a massa molar). Logo, a análise proposta neste trabalho consiste numa ferramenta fácil e eficiente para a otimização do tempo reacional, expondo uma nova abordagem na análise do processo ATRP.

\section{NOMENCLATURA}

$\delta_{2} \quad$ Momento de ordem "dois" para o polímero dormente, mol L ${ }^{-1}$.

$\lambda_{22} \quad$ Momento de ordem "dois" para o polímero morto por combinação, mol L ${ }^{-1}$.

$\lambda_{21} \quad$ Momento de ordem "dois" para o polímero morto via desproporcionamento e/ou transferência de cadeia, mol L ${ }^{-1}$.

$\mu_{2} \quad$ Momento de ordem "dois" para o polímero vivo, $\mathrm{mol} \mathrm{L}^{-1}$.

$\delta_{1} \quad$ Momento de ordem "um" para o polímero dormente, $\mathrm{mol} \mathrm{L}^{-1}$.

$\lambda_{12} \quad$ Momento de ordem "um" para o polímero morto por combinação, mol L" .

$\lambda_{11} \quad$ Momento de ordem "um" para o polímero morto via desproporcionamento e/ou transferência de cadeia, mol L ${ }^{-1}$.

$\mu_{1} \quad$ Momento de ordem "um" para o polímero vivo, $\mathrm{mol} \mathrm{L}^{-1}$.

$\delta_{0} \quad$ Momento de ordem "zero" para o polímero dormente, mol L"

$\lambda_{02} \quad$ Momento de ordem "zero" para o polímero morto por combinação, mol L"-

$\lambda_{01} \quad$ Momento de ordem "zero" para o polímero morto via desproporcionamento e/ou transferência de cadeia, mol $\mathrm{L}^{-1}$.

$\mu_{0} \quad$ Momento de ordem "zero" para o polímero vivo, $\mathrm{mol} \mathrm{L}^{-1}$.

\section{AGRADECIMENTOS}

Os autores deste trabalho agradecem à Petrobras pelo auxílio financeiro concedido.

\section{REFERÊNCIAS}

AL-HARTHI, M.; CHENG, L. S.; SOARES, J. B. P.; SIMON, L. C. Atom-Transfer Radical Polymerization of Styrene With Bifunctional and Monofunctional Initiators: Experimental and Mathematical Modeling Results. J. Polym. Sci., v. 45, p. 2212-2224, 2007.

BADRI, A.; WHITTAKER, M. R.; ZETTERLUND, P. B. Modification of graphene/graphene oxide with polymer brushes using controlled/living radical polymerization. J. Polym. Sci. Part A Polym. Chem., v. 50, n. 15, p. 2981-2992, 2012.

DESTARAC, M. Controlled Radical Polymerization: Industrial Stakes, Obstacles and Achievements. Macromol. React. Eng., v. 4, n. 3-4, p. 165-179, 2010.

FU, Y.; MIRZAEI, A.; CUNNINGHAM, M. F.; HUTCHINSON, R. A. Atom-Transfer Radical Batch and Semibatch Polymerization of Styrene. Macromol. React. Eng., v. 1, n. 4, p. 425-439, 2007. 
GOLDMANN, A. S.; GLASSNER, M.; INGLIS, A. J.; BARNER-KOWOLLIK, C. Postfunctionalization of polymers via orthogonal ligation chemistry. Macromol. Rapid Commun., v. 34, n. 10, p. 810-49, 2013.

HINDMARSH, A. C. ODEPACK: A Systematized Collection of ODE Solvers. Sci. Comput., v. 1, p. 55-64, 1983.

MATYJASZEWSKI, K. Atom Transfer Radical Polymerization: From Mechanisms to Applications. Isr. J. Chem., v. 52, n. 3-4, p. 206-220, 2012.

OHNO, K.; GOTO, A.; FUKUDA, T.; XIA, J.; MATYJASZEWSKI, K. Kinetic Study on the Activation Process in an Atom Transfer Radical Polymerization. Macromolecules, v. 31, p. 2699-2701, 1998.

PHAM, P. D.; MONGE, S.; LAPINTE, V.; RAOUL, Y.; ROBIN, J. J. Various radical polymerizations of glycerol-based monomers. Eur. J. Lipid Sci. Technol., v. 115, n. 1, p. 28-40, 2013.

PORRAS, C. T.; D'HOOGE, D. R.; VAN STEENBERGE, P. H. M.; REYNIERS, M.-F.; MARIN, G. B. A Theoretical Exploration of the Potential of ICAR ATRP for One and Two-Pot Synthesis of Well-Defined Diblock Copolymers. Macromol. React. Eng., v. 7, n. 7, p. 311-326, 2013.

RAY, W. H. On the Mathematical Modeling of Polymerization Reactors. J. Macromol. Sci. Part C Polym. Rev., v. 8, n. 1, p. 1-56, 1972.

SALIAN, V. D.; BYRNE, M. E. Living Radical Polymerization and Molecular Imprinting: Improving Polymer Morphology in Imprinted Polymers. Macromol. Mater. Eng., v. 298, n. 4, p. 379-390, 2013.

SALZINGER, S.; RIEGER, B. Rare Earth metal-mediated group transfer polymerization of vinylphosphonates. Macromol. Rapid Commun., v. 33, n. 16, p. 1327-45, 2012.

SINGH, P.; SRIVASTAVA, A.; KUMAR, R. Synthesis of amphiphilic poly(Nvinylcaprolactam) using ATRP protocol and antibacterial study of its silver nanocomposite. J. Polym. Sci. Part A Polym. Chem., v. 50, n. 8, p. 1503-1514, 2012.

TANG, W.; MATYJASZEWSKI, K. Effects of Initiator Structure on Activation Rate Constants in ATRP. Macromolecules, v. 40, n. 6, p. 1858-1863, 2007.

VIEIRA, R. P.; OSSIG, A.; PEREZ, J. M.; GRASSI, V. G.; PETZHOLD, C. L.; PERES, A.; COSTA, J. M.; LONA, L. M. F. Simulation of the Equilibrium Constant Effect on the Kinetics and Average Properties of Polystyrene Obtained by ATRP. J. Braz. Chem. Soc., v. 24, n. 12, p. 2008-2014, 2013.

ZHANG, M.; RAY, W. H. Modeling of living free-radical polymerization processes. I. Batch, semibatch, and continuous tank reactors. J. Appl. Polym. Sci., v. 86, n. 7, p. 1630-1662, 2002.

ZHAO, M.; ZHANG, H.; MA, F.; ZHANG, Y.; GUO, X.; ZHANG, H. Efficient synthesis of monodisperse, highly crosslinked, and "living" functional polymer microspheres by the ambient temperature iniferter-induced "living" radical precipitation polymerization. $J$. Polym. Sci. Part A Polym. Chem., v. 51, n. 9, p. 1983-1998, 2013.

ZHOU, Y. N.; LI, J. J.; LUO, Z. H. Synthesis of gradient copolymers with simultaneously tailor-made chain composition distribution and glass transition temperature by semibatch ATRP: From modeling to application. J. Polym. Sci. Part A Polym. Chem., v. 50, n. 15, p. 3052-3066, 2012.

ZHU, S. Modeling of molecular weight development in atom transfer radical polymerization. Macromol. Theory Simulations, v. 8, n. 1, p. 29-37, 1999. 